The Genus Lembosia on Humboldtia species in Kerala, India

\author{
V. B. Hosagoudar*, A. Sabeena and Jacob Thomas \\ Tropical Botanic Garden and Research Institute, Palode 695 562, Thiruvananthapuram, Kerala \\ vbhosagoudar@rediffmail.com*
}

Abstract: The genus Humboldtia comprises six species and of which five are endemic to Peninsular India. Of these, Humboldtia unijuga and $H$. vahliana were infected with three species of the genus Lembosia, namely, Lembosia humboldtiae, L. humboldtiicola and L. humboldtiigena. The former species was described from Kerala State and the latter two are described here as new to science. All these species are described and illustrated in detail to provide the consolidated account of the species known on this host genus.

Keywords: Fungi, Lembosia, Humboldtia, Kerala, India, new species.

Introduction

The genus Humboldtia (Authority?) is an IndoSri Lankan plant, comprises six species, of which five are known in India and are endemic to southern Western Ghats. These are mostly distributed in the evergreen forests at an altitude from 150-1250 m above msl. Of these, Humboldtia unijuga Bedd. and $H$. vahliana Wight were found infected with Lembosia species.

The genus Lembosia (Authority?), a type genus of the family Lembosiaceae, represents 144 species in the world (Song \& Hosagoudar, 2003), characterized by the brown, septate mycelium with

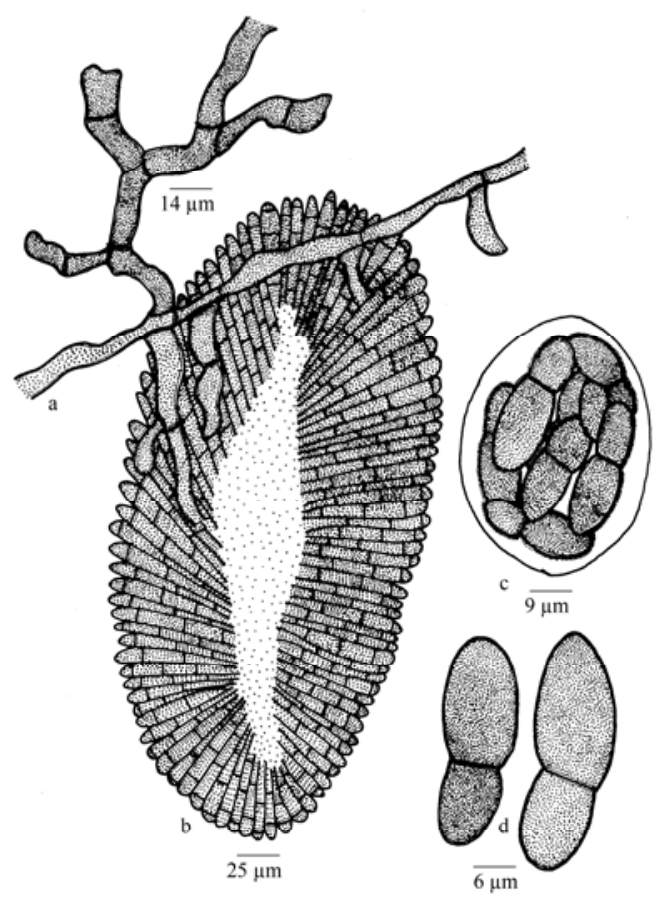

Fig. 1. Lembosia humboldtiae Hosag. \& Abraham a. Appressoriate mycelium, b. Thyriothecium, c. Ascus, d. Ascospores laterally placed appressoria; ovate, elongated to furcate thyriothecia dehisce longitudinally at the centre and asci ovate to globose with 4-8 brown uniseptate ascospores.

Taxonomy

Key to the species of the genus Lembosia on Humboldtia species

1.Appressoria oblong to elongated, 1-3 celled, straight, flexuous to crooked, cells of ascospores unequal ... L. humboldtiiae

1. Appressoria and ascospores not so $\quad . .2$

2. Appressoria opposite, ovate to conoid ... L. humboldtiicola

2. Appressoria alternate, globose

$$
\text { ... L. humboldtiigena }
$$

\section{Descriptions to the species}

1. Lembosia humboldtiae Hosag. \& Abraham, Mycol. Res. 102: 186, 1996 (humboltiae)

Colonies amphigenous, dense, crustose, up to $5 \mathrm{~mm}$ in diameter, confluent. Hyphae flexuous to crooked, branching opposite at acute to wide angles, closely reticulate, cells 15-44 x 2-4 $\mu \mathrm{m}$. Appressoria scattered, some part of the mycelium devoid of appressoria, alternate to opposite,
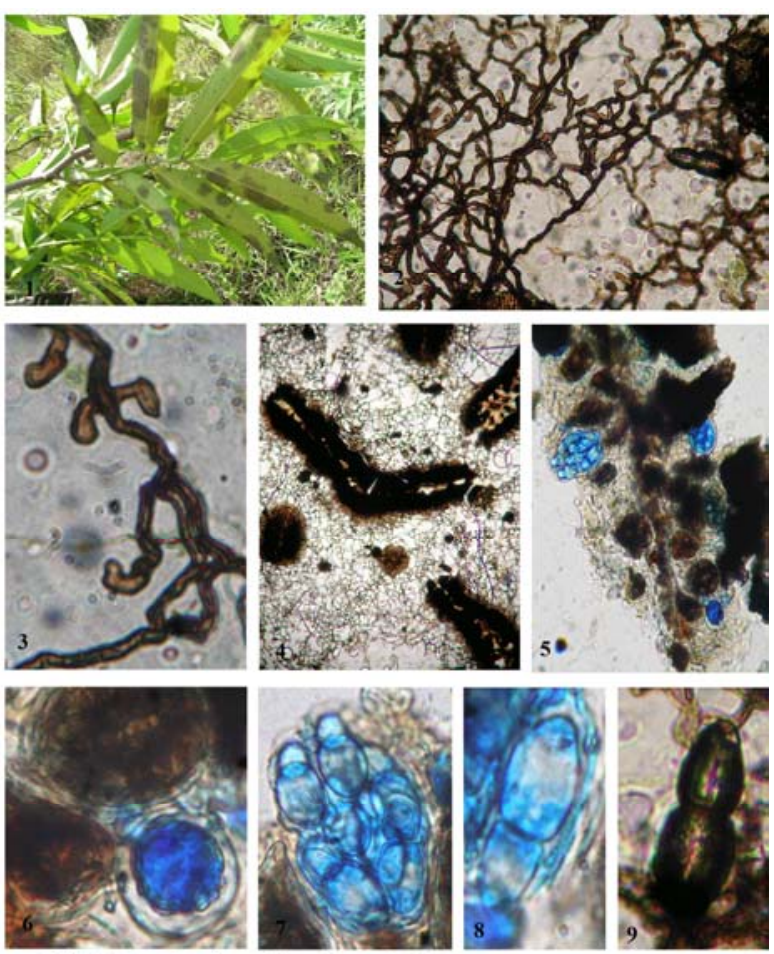

PLATE-1. Lembosia humboldtiae Hosag. \& Abraham

1. Infected leaves, 2, Mycelium, 3, Mycelium with opposite and many celled appresson

4. Thyriothecia, 5. Broken thyriothecium with exposed asci, 6. Ascus, 7. Ascus having ascospores showing unequal cells, 8-9. Ascospores having unequal cells. 
straight, curved, flexuous to crooked, 1-3 celled, 11-44 $\mu \mathrm{m}$ long; stalk cells 1-2 septate, 12-22 $\mu \mathrm{m}$ long; head cells ovate, straight, curved, entire to rarely lobate, 6-22 x 2-4 $\mu \mathrm{m}$. Thyriothecia scattered, rounded to elongated, longitudinally
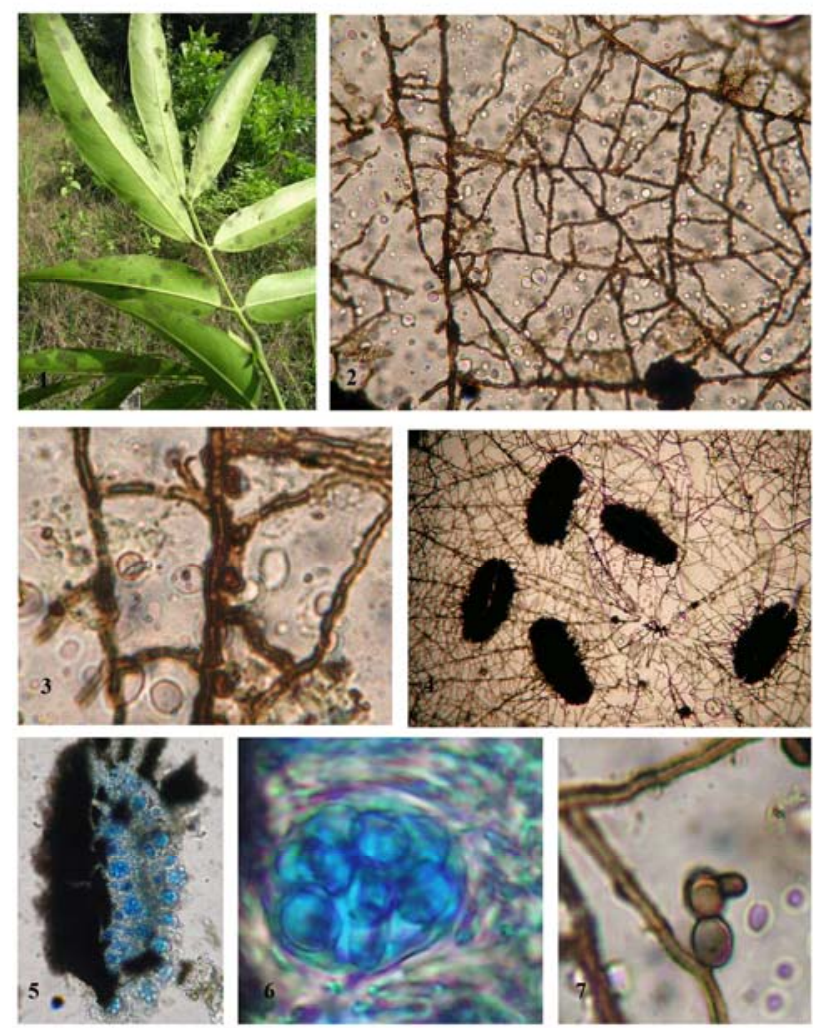

PLATE-2, Lembosia humboldtiigena sp. nov

1. Infected leaves, 2. Branched mycelium, 3. Appressoriate mycelium,

4. Elongated thyriothecia in the colony, 5. Dehisced thyriothecium with exposed asci, 6. Ascus, 7. Ascospore.

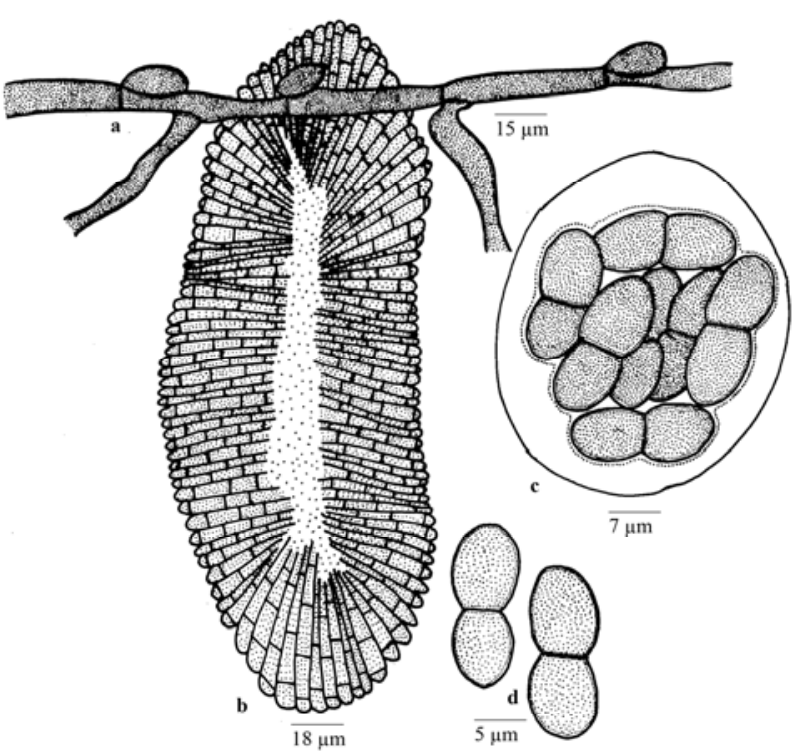

Fig. 2. Lembosia humboldtiigena sp. nov. a. Appressoriate mycelium, b. Thyriothecium, c. Ascus, d. Ascospores fissured at the centre, $200-1000 \times 180-250 \mu \mathrm{m}$, margin crenate to fimbriate, fringed hyphae flexuous to crooked; asci octosporous, subglobose to ovoid, 22-42 $\mu \mathrm{m}$ in diameter; ascospores 1septate, brown, constricted at the septum, 22-35 $\mu \mathrm{m}$ long, lower cell smaller, ovate, $9-15 \times 6$ -
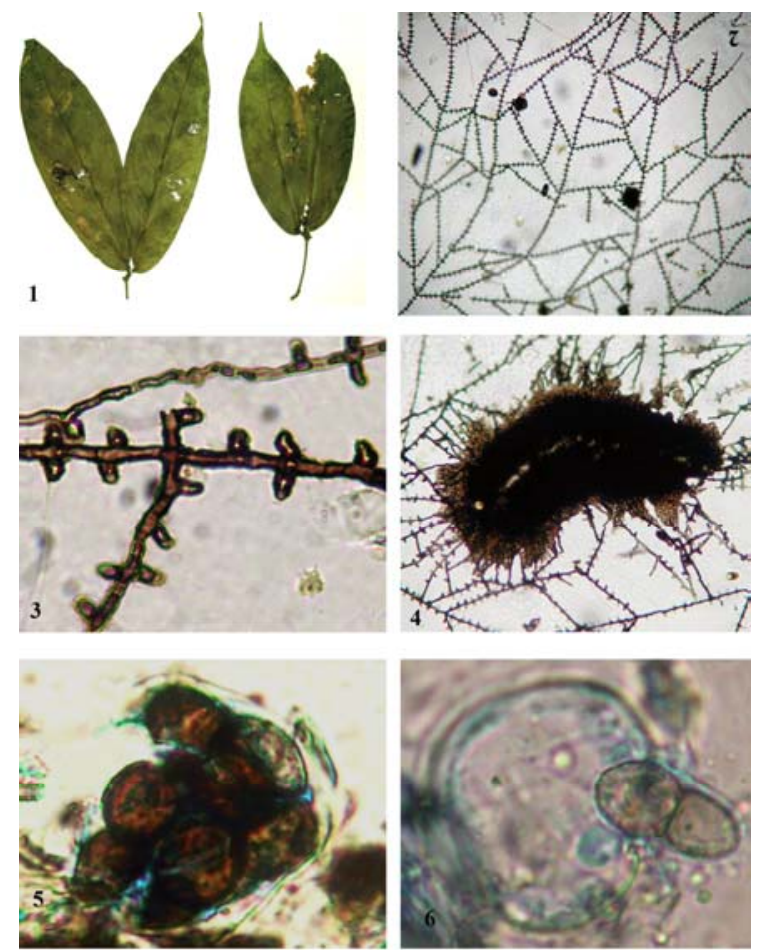

PLATE-3. Lembosia humboldtiicola sp. nov.

1. Infected leaves, 2, Mycelium, 3. Appressoriate mycelium,

4. Thyriothecium, 5. Broken ascus, 6. Ascospore emerging from the ascus.

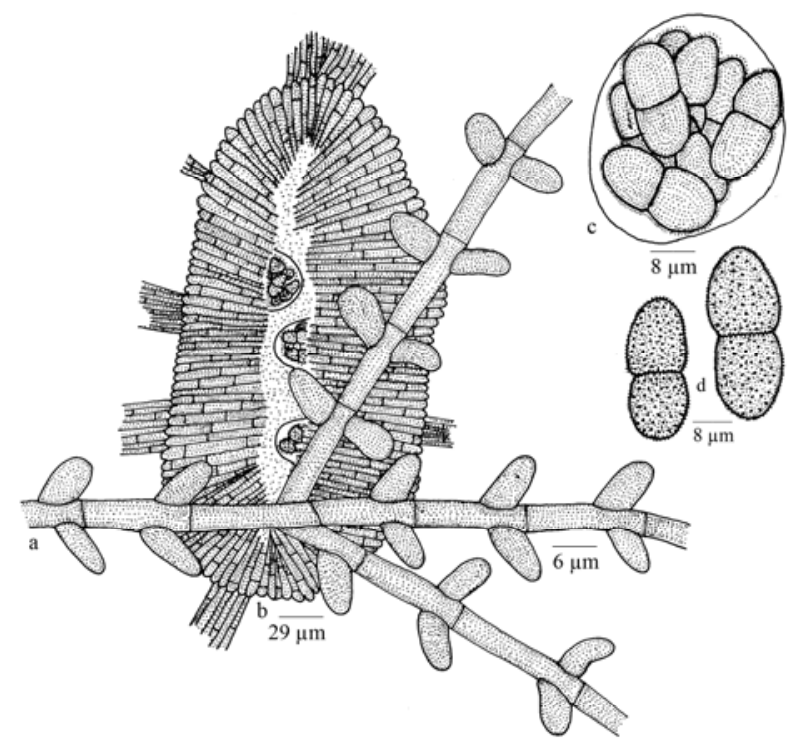

Fig. 3. Lembosia humboldtiicola sp. nov. a. Appressoriate mycelium, b. Thyriothecium, c. Ascus, d. Ascospores 
$11 \mu \mathrm{m}$, upper cell larger than the lower, ovate, 13$20 \times 11-13 \mu \mathrm{m}$, wall smooth.

Materials examined. On leaves of Humboldtia vahliana Wight (Caesalpiniaceae), Kombe, Meenmutty, Neyyar Wildlife Sanctuary, Thiruvananthapuram, Kerala, March 9, 1996, V.B. Hosagoudar $\mathrm{HClO} 42179$ (type), TBGT 51 (isotype); Humboldtia sp., TBGRI Campus, Palode, Thiruvananthapuram, Kerala, Nov. 25, 2000, T. Sabu HCIO 43829, TBGT 379; Dec. 28, 2006, A. Sabeena \& M.C. Riju HCIO, TBGT 3008; Kulamavu, Idukki, Kerala, Sept. 5, 2001, M. Kamarudeen HCIO 45109, TBGT 1164; Arboretum, TBGRI Campus, Palode, Thiruvananthapuram, Nov. 4, 2004, T. Sabu HClO 47318, TBGT 2356; near Peppara Dam, Peppara Wildlife Sanctuary, Nov. 18, 2007, Jacob Thomas and Vimalkumar TBGT 3009. This species is distinct from rest of the two in having unicellular to multi cellular, flexuous to crooked stalk cells of the appressoria and unequal cells in the ascospores (Hosagoudar \& Abraham, 1998).

\section{Lembosia humboldtiigena sp. nov.}

Coloniae amphigenae, densae, ad $5 \mathrm{~mm}$ diam., confluentes. Hyphae subrectae vel flexuosae, alternate vel irregulariter acuteque vel laxe ramosae, laxe vel arte reticulatae, cellulae 44$55 \times 3-5 \mu \mathrm{m}$. Appressoria dispersa, alternata vel unilateralis, unicellularis, crassa posita, ovata, globosa, integra vel sublobata, 2-9 x 2-6 $\mu \mathrm{m}$. Thyriothecia dispersa, rotundata vel elongata, dehiscentes longitudinalis ad centro, 150-480 x 100-330 $\mu \mathrm{m}$, margine crenatae vel fimbriatae, hyphae fringiorae flexuosae, exappressoriatae; asci sub globosi, octospori, 31-44 $\mu \mathrm{m}$ diam.; ascosporae hyalinae ad initio, brunneae ad maturitatae, 1-septatae, conglobatae, constrictae ad septatae, 13-17 x 4-9 $\mu \mathrm{m}$, parietus glabrus.

Colonies amphigenous, dense, up to $5 \mathrm{~mm}$ diameter, confluent. Hyphae substraight to flexuous, branching alternate to irregular at acute to wide angles, loosely to closely reticulate, cells 44-55 x 3-5 $\mu \mathrm{m}$. Appressoria scattered, alternate to unilateral, unicellular, broad based, ovate, globose, entire to sublobate, 2-9 x 2-6 $\mu \mathrm{m}$. Thyriothecia scattered, rounded to elongated, longitudinally fissured at the centre, $150-480 \times 100$ $330 \mu \mathrm{m}$, margin crenate to fimbriate, fringed hyphae flexuous, exappressoriate; asci sub globose, octosporous, 31-44 $\mu \mathrm{m}$ in diameter; ascospores initially hyaline, brown at maturity, 1septate, conglobate, constricted at the septum, 13$17 \times 4-9 \mu \mathrm{m}$, wall smooth.

Materials examined. On leaves of Humboldtia vahliana Wight (Caesalpiniaceae), TBGRI Campus, Palode, Thiruvananthapuram, Kerala, India, Dec. 28, 2006, A. Sabeena and M.C. Riju
TBGT 3008 (type). Unicellular, scattered, ovate, globose, entire to sublobate appressoria distinguishes this from the other two species.

\section{Lembosia humboldtiicola sp. nov.}

Coloniae hypophyllae, tenues, crustosae, ad 2 mm diam., confluentes. Hyphae rectae vel subrectae, opposite vel irregulariter acuteque vel laxe ramosae, laxe reticulatae, cellulae 9-14 x 2-5 $\mu \mathrm{m}$. Appressoria plerumque opposita, subopposita, paucae unilateralis, unicellularis, ovata, conoidea vel cylindracea, crassa posita, sessilis, integra, 5-7 x 4-10 $\mu \mathrm{m}$. Thyriothecia dispersa, ad intio rotundata, elongata ad maturitata, dehiscentes longitudinalis ad centro, 250-400 x 150-200 $\mu \mathrm{m}$, margine crenatae vel fimbriatae, hyphae fringiorae subrectae vel flexuosae, exappressoriatae; asci octospori, globosi, ad $48 \mu \mathrm{m}$ diam.; ascosporae hyalinae ad initio, fortiter brunneae ad maturitatae, uniseptatae, conglobatae, constrictae ad septatae, 31-36 x 11$13 \mu \mathrm{m}$, parietus echinulatus.

Colonies hypophyllous, thin, crustose, up to 2 $\mathrm{mm}$ in diameter, confluent. Hyphae straight to substraight, branching opposite to irregular at acute to wide angles, loosely reticulate, cells 9-14 x 2-5 $\mu \mathrm{m}$. Appressoria mostly opposite, sub opposite, few unilateral, unicellular, ovate, conoid to cylindrical, broad based, sessile, entire, 5-7 x 4$10 \mu \mathrm{m}$. Thyriothecia scattered, initially rounded, elongated at maturity, longitudinally dehisced at the centre, $250-400 \times 150-200 \mu \mathrm{m}$, margin crenate to fimbriate, fringed hyphae substraight to flexuous, appressoriate; asci octosporous, globose, up to 48 $\mu \mathrm{m}$ diameter; ascospores initially hyaline, deep brown at maturity, uniseptate, conglobate, constricted at the septum, 31-36 x 11-13 $\mu \mathrm{m}$ long, wall echinulate.

Materials examined: On leaves of Humboldtia unijuga Bedd. (Caesalpiniaceae), Athirumala, Peppara Wildlife Sanctuary, Thiruvananthapuram, Kerala, India, Feb. 28, 2008, Jacob Thomas et al. TBGT 3010 (type). Opposite to sub opposite, ovate to conoid appressoria distinguishes this species from the other two species.

\section{Acknowledgement}

We are grateful to the Director, Tropical Botanic Garden and Research Institute (TBGRI), Palode for the facilities and to Forest Department, Govt. of Kerala for the permission to collect the materials.

\section{Reference}

1. Hosagoudar VB and Abraham TK (1998) Four new foliicolous Ascomycetes from Kerala, India. Mycol. Res. 102, 184-86.

2. Song B and Hosagoudar VB (2003) A list of Lembosia species based on the literature. Guizhou Science 21, 93-101. 